\title{
The antitumor activity of a doxorubicin loaded, iRGD-modified sterically-stabilized liposome on BI6-FI0 melanoma cells: in vitro and in vivo evaluation
}

\author{
This article was published in the following Dove Press journal: \\ International Journal of Nanomedicine \\ 12 July 2013 \\ Number of times this article has been viewed
}

Ke-Fu Yu'
Wei-Qiang Zhang'
Li-Min Luo'
Ping Song'
Dan Li'
Ruo Du'
Wei Ren'
Dan Huang'
Wan-Liang Lu',2
Xuan Zhang'
Qiang Zhang',2
'Department of Pharmaceutics, School
of Pharmaceutical Sciences, Peking
University, Beijing, People's Republic
of China; ${ }^{2}$ State Key Laboratory of
Natural and Biomimetic Drugs, School
of Pharmaceutical Sciences, Peking
University, Beijing, People's Republic
of China

of China

\begin{abstract}
Considering the fact that iRGD (tumor-homing peptide) demonstrates tumor-targeting and tumor-penetrating activity, and that B16-F10 (murine melanoma) cells overexpress both $\alpha \mathrm{V}$ integrin receptor and neuropilin-1 (NRP-1), the purpose of this study was to prepare a novel doxorubicin (DOX)-loaded, iRGD-modified, sterically-stabilized liposome (SSL) (iRGD-SSLDOX) in order to evaluate its antitumor activity on B16-F10 melanoma cells in vitro and in vivo. The iRGD-SSL-DOX was prepared using a thin-film hydration method. The characteristics of iRGD-SSL-DOX were evaluated. The in vitro leakage of DOX from iRGD-SSL-DOX was tested. The in vitro tumor-targeting and tumor-penetrating characteristics of iRGD-modified liposomes on B16-F10 cells were investigated. The in vivo tumor-targeting and tumor-penetrating activities of iRGD-modified liposomes were performed in B16-F10 tumor-bearing nude mice. The antitumor effect of iRGD-SSL-DOX was evaluated in B16-F10 tumor-bearing C57BL/6 mice in vivo. The average particle size of the iRGD-SSL-DOX was found to be $91 \mathrm{~nm}$ with a polydispersity index (PDI) of 0.16. The entrapment efficiency of iRGD-SSL-DOX was $98.36 \%$. The leakage of DOX from iRGD-SSL-DOX at the 24-hour time point was only 7.5\%. The results obtained from the in vitro flow cytometry and confocal microscopy, as well as in vivo biodistribution and confocal immunofluorescence microscopy experiments, indicate that the tumor-targeting and tumor-penetrating activity of the iRGD-modified SSL was higher than that of unmodified SSL. In vivo antitumor activity results showed that the antitumor effect of iRGD-SSL-DOX against melanoma tumors was higher than that of SSL-DOX in B16-F10 tumor-bearing mice. In conclusion, the iRGD-SSL-DOX is a tumor-targeting and tumor-penetrating peptide modified liposome which has significant antitumor activity against melanoma tumors.
\end{abstract}

Keywords: tumor-targeting and tumor-penetrating, integrin receptor, NRP-1, iRGD, liposome, doxorubicin

\section{Introduction}

Tumor-targeted drug delivery systems containing antitumor agents are believed to have opened a new era in traditional chemotherapy. ${ }^{1-3}$ Ligand-modified targeting drug delivery systems can bind with specific tumor surfaces' overexpressing receptors to increase the drug concentration at the tumor site and significantly improve therapeutic efficacy. ${ }^{4,5}$ For example, Arg-Gly-Asp (RGD) - or Asn-Gly-Arg (NGR)-modified targeting drug delivery systems have been extensively investigated. ${ }^{6-10}$

Recently, a tumor-targeting and tumor-penetrating cyclic peptide, tumor-homing peptide iRGD (peptide with Cys-Arg-Gly-Asp-Lys/Arg-Gly-Pro-Asp/Glu-Cys
Correspondence: Xuan Zhang School of Pharmaceutical Sciences, Peking University,

Xueyuan Road 38, Beijing, 100191, People's Republic of China

Tel +86 I0 82802683

Fax +861082802683

Email xuanzhang@bjmu.edu.cn 
[CRGDK/RGPD/EC]), has been reported to enhance vascularity and tissue permeability in a tumor-specific and neuropilin-1 (NRP-1)-dependent manner. ${ }^{11}$ The mechanism for how iRGD homes to tumor sites has been explained in detail. ${ }^{11,12}$ Briefly, iRGD targets tumors by first binding to $\alpha \mathrm{V}$ integrins and then being proteolytically cleaved in the tumor to produce CRGDK/R, which has an affinity for NRP-1, in order to trigger tissue penetration. Thus, when nanoparticles, nanogels, or anticancer agents are modified with iRGD, their tissue-penetrating and tumor-targeting properties can be improved. ${ }^{11,13-17}$ Additionally, when iRGD was coadministrated with drugs or a systemic injection drug delivery system, antitumor activities were also improved. ${ }^{12}$

In the past, liposomes have been used as drug carriers to improve the pharmacokinetics of drugs, resulting in reduced toxicities and enhanced therapeutic efficacies. ${ }^{18}$ However, conventional liposomes have reported rapid uptake and accumulation by phagocytic cells of the mononuclear phagocyte system (also know as the reticuloendothelial system [RES]), after systemic administration. ${ }^{19}$ PEG polyethylene glycol (PEGylation) modification prevents the recognition of conventional liposomes by opsonins and therefore reduces their clearance by cells of the RES. ${ }^{20}$ Therefore, PEGylated liposomes are often referred to as sterically stabilized liposomes (SSL). Using PEGylated phospholipids, the apparent terminal half-life of a liposome can be extended. ${ }^{21}$ In addition, SSL could spontaneously accumulate in solid tumors via the enhanced permeability and retention (EPR) effect, presenting passive targeting activity. ${ }^{22,23}$

Considering the fact that iRGD has a tumor-targeting and tumor-penetrating effect as well as the fact that some tumor cells overexpress both the $\alpha \mathrm{v}$ intergrin receptor and NRP-1, in the present study we selected iRGD as a targeting ligand, SSL as a delivery system, and doxorubicin hydrochloride (DOX) as a model drug to prepare a DOX-loaded, iRGD-modified SSL (iRGD-SSL-DOX). Due to overexpression of $\alpha \mathrm{v}$ integrin receptor and NRP-1, ${ }^{24,25}$ the B16-F10 cells were selected as the tumor cell model. The antitumor activity of iRGD-SSL-DOX on B16-F10 cells was investigated in vitro and in vivo.

\section{Materials and methods Materials}

1,2-Distearoyl-sn-Glycero-3-Phosphoethanolamine-N[Maleimide(polyethylene-Glycol)-2000] (DSPE-PEG-MAL) and 1,2-distearoyl-sn-glycero-3-Phosphoethanolamine-N [methoxy(polyethylene glycol)-2000] (DSPE-PEG) were purchased from Avanti Polar Lipids (Alabaster, AL, USA). Coumarin-6 and cholesterol were obtained from
Sigma-Aldrich (St Louis, MO, USA). iRGD peptide (CRGDKGPDC) was synthesized by GL Bio-Chem Co, Ltd (Shanghai, People's Republic of China). DOX was supplied by Zhejiang Hisun Pharmaceutical Co, Ltd (Taizhou Zhejiang, People's Republic of China). Near infrared lipophilic carbocyanine dye 1,1'-dioctadecyltetramethyl indotricarbocyanine iodide (DiR) and the fluorescent probe, 1,1'-dioctadecyl-3,3,3',3'-tetramethylindocarbocyanine perchlorate (DiI) were obtained from Biotium Inc (Hayward, CA, USA). Hoechst 33258 and Fluorescein Isothiocyanate (FITC) goat anti-rabbit secondary antibodies (1:1000) were obtained from Molecular Probes Inc (Eugene, OR, USA). Rabbit polyclonal to CD31 $(10 \mu \mathrm{g} / \mathrm{mL})$ and rabbit polyclonal to fibrinogen $(10 \mu \mathrm{g} / \mathrm{mL})$ were purchased from Abcam Inc (Cambridge, Massachusetts, USA). Cell culture media, DMEM (Dulbecco's Modified Eagle Medium, high glucose), RPMI 1640 (Roswell Park Memorial Institute 1640), penicillin-streptomycin, fetal bovine serum (FBS), and L-glutamine, were obtained from GIBCO Invitrogen Corp (Life Technologies, Carlsbad, CA, USA). All other reagents were of analytical grade.

\section{Cells}

Murine B16-F10 cell line was obtained from Chinese Academy of Sciences Cells Bank (Shanghai, People's Republic of China). The cells were grown in DMEM medium (high glucose) supplemented with 10\% FBS and 1\% glutamine penicillin-streptomycin at $37^{\circ} \mathrm{C}$ and $5 \% \mathrm{CO}_{2}$. The human breast adenocarcinoma MCF-7 cell line was supplied by the Institute of Basic Medical Sciences (Chinese Academy of Medical Sciences, Beijing, People's Republic of China) and cultivated according to the recommended instructions.

\section{Animals}

Female BALB (Bagg Albino)/c nude mice weighing 20-25 g (5-6 weeks old) and female C57BL/6 mice weighing 20-24 g (5-6 weeks old) were supplied by the Experimental Animal Center of Peking University Health Science Center (Beijing, People's Republic of China). The temperature and relative humidity were maintained at $25^{\circ} \mathrm{C}$ and $45 \%-55 \%$, respectively. All care and handling of the animals was performed in accordance with the requirements of the Institutional Authority for Laboratory Animal Care of Peking University.

\section{Synthesis of iRGD-PEG-DSPE}

iRGD-PEG-DSPE was synthesized from iRGD and DSPE-PEG-maleimide in a single step that coupled iRGD to DSPE-PEG using a method previously reported 
by our laboratory. ${ }^{9,10,26}$ In brief, DSPE-PEG-maleimide mixed with $i R G D$ at a $1: 1$ molar ratio (iRGD:DSPEPEG-maleimide $=1: 1)$ in Hepes $(\mathrm{pH}=6.5)$. This reaction mixture was gently stirred at room temperature for 48 hours under nitrogen gas. After that, the resulting reaction mixture was placed in a dialysis bag (molecular weight cutoff $=3500$ $\mathrm{Da}$ ) and dialyzed in deionized water for 48 hours to remove the free iRGD. The final solution in the dialysis bag was lyophilized and stored at $-20^{\circ} \mathrm{C}$ until used.

\section{Preparation of iRGD-SSL-DOX}

The iRGD-SSL-DOX were prepared by a thin-film hydration method. Briefly, egg phosphatidylcholine, cholesterol, DSPE-PEG, iRGD-PEG-DSPE (65:30:4:1 molar ratio) were dissolved in chloroform. The solvent was evaporated using an RE52 rotary evaporator (Shanghai Yarong Biochemistry Instrument Company, Shanghai, People's Republic of China) in a round-bottomed flask at $45^{\circ} \mathrm{C}$ for about 40 minutes to form a solid film. Next, this film was flushed with nitrogen gas for 30 minutes and stored overnight in a desiccator to remove any traces of chloroform. A volume of $2 \mathrm{~mL} 300 \mathrm{mM}$ citric acid buffer $(\mathrm{pH}=4.0)$ was added in the flask and sonicated in a bath sonicator for 30 minutes to form liposomes. The resulting liposomes were then extruded eleven times through $100 \mathrm{~nm}$ polycarbonate membrane filters using a mini-extruder (Avanti Polar Lipids Inc, Alabaster, AL, USA). The obtained liposomes were then passed through a Sephadex G-50 (Sigma-Aldrich, St Louis, MO, USA) column to remove the external citric acid of the liposomes with a $20 \mathrm{mM}$ Hepes buffer solution (HBS) containing $150 \mathrm{mM} \mathrm{NaCl}$ (HBS, $\mathrm{pH}=7.4$ ). DOX solution was added to the liposomes at a DOX/lipid (weight/weight) ratio of 1:20 to load into these blank liposomes using a pH-gradient method. The mixed solution was incubated for 1 hour at $37^{\circ} \mathrm{C}$ and then passed through a Sephadex G-50 column to remove the unentrapped DOX with HBS.

The unmodified SSL containing DOX (SSL-DOX) were prepared according to the above procedure except that the equivalent molar amount of iRGD-PEG-DSPE was replaced by DSPE-PEG.

The coumarin-6, DiR or DiI loaded SSL (iRGD-SSLcoumarin-6, and SSL-coumarin-6, iRGD-SSL-DiR and SSL-DiR, iRGD-SSL-DiI and SSL-DiI) were also prepared by the thin-film hydration method.

\section{Characterization of iRGD-SSL-DOX}

For particle size analysis, the final concentration of DOX in SSL-DOX or iRGD-SSL-DOX was about $0.3 \mathrm{mg} / \mathrm{mL}$. The particle size and zeta potential of iRGD-SSL-DOX were measured by using a Malvern Zetasizer Nano-ZS (Malvern Instruments Ltd, Malvern, Worcestershire, UK) at $25^{\circ} \mathrm{C}$. The liposomal encapsulation efficiency was determined as described below. Briefly, the final liposomes were passed through a Sephadex G-50 column to remove free DOX, followed by disruption with $10 \%$ Triton X-100 (volume to volume $[\mathrm{v} / \mathrm{v}])$; the DOX in the liposomes was then measured in a spectrofluorometer (RF-5301PC; Shimadzu Corp, Nakagyo-ku, Kyoto, Japan). In addition, the same amount of liposomes was treated as above, except they were passed through a Sephadex G-50 column to obtain the total concentration of DOX. The encapsulation efficiency was calculated by the following formula:

\section{Encapsulation efficiency $=\frac{\text { DOX concentration in the filtered liposomes }}{\text { DOX concentration in the unfiltered liposomes }} \times 100$ \\ In vitro leakage of DOX from iRGD-SSL- DOX}

The leakage of DOX from iRGD-SSL-DOX was tested using a dialysis method. In brief, iRGD-SSL-DOX (1.0 mL, $0.15 \mathrm{mg} / \mathrm{mL}$ ) was placed in a dialysis bag (MWCO [Molecular Weight Cut-Off] 8,000-14,000). The dialysis bag was then immersed in $20 \mathrm{~mL}$ release medium (phosphate buffered saline $[\mathrm{PBS}][\mathrm{pH}=7.4]$ ) and incubated in an orbital shaker for 120 hours at $37^{\circ} \mathrm{C}$. A collection of $1.0 \mathrm{~mL}$ samples was then taken out from the release medium at the predetermined time intervals, and a similar volume of fresh medium was added. The concentration of DOX was determined using a spectrofluorometer (RF-5301PC). The excitation and emission wavelengths were set at $494 \mathrm{~nm}$ and $591 \mathrm{~nm}$, respectively. DOX release was measured for 120 hours. After the last sample in the medium was taken out at the 120-hour time point, solution in dialysis bag was mixed with the release medium; a volume of $10 \mu \mathrm{L}$ of $10 \%(\mathrm{v} / \mathrm{v})$ Triton X-100 was then added in the released medium. This mixture was determined as a positive control. The percentage DOX release was calculated as $\left(\mathrm{I}_{\mathrm{t}}-\mathrm{I}_{0}\right) /\left(\mathrm{I}_{100}-\mathrm{I}_{0}\right) \times 100 \%$, in which $\mathrm{I}_{\mathrm{t}}$ is the fluorescence at time point $t, I_{0}$ is the fluorescence at the time the dialysis bag immersed in the released medium, and $\mathrm{I}_{100}$ is the fluorescence of the sample after the addition of Triton $\mathrm{X}-100$ at the 120 -hour time point.

\section{Flow cytometry}

B16-F10 cells were seeded at a density of $3 \times 10^{5}$ cells/well in 6-well plates and incubated at $37^{\circ} \mathrm{C}$ for 24 hours. After 24 hours incubation, the medium was replaced with SSL-coumarin-6, 
iRGD-SSL-coumarin-6 or coumarin-6 solution (the final concentration of coumarin- 6 was $150 \mathrm{ng} / \mathrm{mL}$ ). The plates were divided in two groups (with or without trypsin treatment). For group 1 (without trypsin treatment group), after 2 hours incubation at $37^{\circ} \mathrm{C}$, the cells were washed three times with PBS solution. For group 2 (with trypsin treatment group), SSL-coumarin-6, iRGD-SSL-coumarin-6 or coumarin-6 solution (the final concentration of coumarin-6 was $150 \mathrm{ng} / \mathrm{mL}$ ) received $50 \mu \mathrm{L}$ trypsin solution $(250 \mu \mathrm{g} / \mathrm{mL})$ and was incubated for 5 minutes at $37^{\circ} \mathrm{C}$; the soybean inhibitor $(50 \mu \mathrm{L}, 30 \mathrm{mg} / \mathrm{mL})$ was then added to stop the reaction. The above solutions were added and incubated with cells for 2 hours at $37^{\circ} \mathrm{C}$. The cells were then washed three times with PBS. All cells in both groups were then harvested by trypsinization and centrifuged at 1,000 rpm for 5 minutes and resuspended in $500 \mu \mathrm{L}$ PBS medium and tested using a FACScan (Becton, Dickinson and Company, Franklin Lakes, NJ, USA). The coumarin-6 in the cells was excited with an argon laser $(467 \mathrm{~nm})$ and fluorescence was detected at $502 \mathrm{~nm}$.

For anther flow cytometry experiment, the MCF-7 cells were seeded at a density of $2 \times 10^{5}$ cells/well in 6-well plates and performed as described above in the B16-F10 cell line.

\section{Confocal microscopy studies}

Following incubation of B16-F10 cells $\left(3 \times 10^{5}\right.$ cells $)$ on glass-bottomed dishes containing culture medium at $37^{\circ} \mathrm{C}$ for 24 hours, cell culture media containing SSL-coumarin-6, iRGD-SSL-coumarin-6 or coumarin-6 solution (final drug concentration $150 \mathrm{ng} / \mathrm{mL}$ ) were added to each dish and incubated for another 2 hours at $37^{\circ} \mathrm{C}$. After the medium was removed, the cells were washed with ice-cold PBS followed by fixing with 4\% paraformaldehyde in PBS; the cells were then processed in a Hoechst 33258 stain for 20 minutes. The fluorescent images of the cells were inspected using a TCS SP2 confocal microscope (Leica Microsystems, Wetzlar, Germany).

\section{In vivo biodistribution imaging}

A volume of $0.1 \mathrm{ml}$ of B16-F10 cells $\left(1 \times 10^{6}\right)$ suspension was administrated via subcutaneous injection into the right armpits of nude female BALB/c nude mice to prepare the B16-F10 tumor-bearing nude mice model. Once the tumor masses in the xenografts reached $200 \mathrm{~mm}^{3}$, a volume of $200 \mu \mathrm{L}$ physiological saline, SSL-DiR or iRGD-SSL-DiR was intravenously (IV) administrated via the tail vein of the tumor-bearing nude mice at a dose of 1,200 ng/mouse. ${ }^{10}$ At the predetermined time points, mice were anaesthetized by isoflurane (1.5\%) and scanned using a Kodak In-Vivo Imaging System FX PRO (Carestream Health, Inc, Rochester, NY, USA) with an excitation bandpass filter at $730 \mathrm{~nm}$ and an emission at $790 \mathrm{~nm}$. The observed time was set at 1 hour, 3 hours, 6 hours, 12 hours, and 24 hours after administration. The exposure time was set at 60 seconds per image. The fluorescent signal intensities in the tumor-bearing nude mice were analyzed using Carestream MI SE software (Carestream Health, Inc). For each near-infrared spectroscopy image, a corresponding X-ray image was taken to identify the anatomical location of the tumor.

\section{Immunohistochemistry}

The tumor-bearing nude mice, prepared as described above, received an IV administration of iRGD-SSL-DiI or SSL-DiI at a dose of $200 \mu \mathrm{g} / \mathrm{kg}$ via the tail vein when the tumor masses reached about $200 \mathrm{~mm}^{3}$ in volume; then, after 3 hours administration, the mice were sacrificed, and the tumors were harvested and frozen in optimal cutting temperature compound embedding medium. The tumor sections $(6 \mu \mathrm{m})$ were incubated with $1 \%$ bovine serum albumin for 3 hours at room temperature, followed by incubation with the primary antibody (rabbit polyclonal to $\mathrm{CD} 31$ or rabbit polyclonal to fibrinogen) overnight at $4{ }^{\circ} \mathrm{C}$; the primary antibodies were then detected with FITC goat anti-rabbit secondary antibodies. Nuclei were counterstained with Hoechst $33258(5 \mu \mathrm{g} / \mathrm{mL})$. The sections were put in Gel Mount ${ }^{\mathrm{TM}}$ mounting medium (Corporation, Foster City, CA, USA) and observed under a confocal microscope (Leica Microsystems).

\section{In vivo anti-tumor activity of iRGD-SSL- DOX}

To prepare the tumor-bearing C57BL/6 mice model, female C57BL/6 mice were inoculated via subcutaneous injection with $0.1 \mathrm{~mL} \mathrm{B16-F10} \mathrm{cell} \mathrm{suspension}\left(1 \times 10^{6}\right)$ in the right armpits. ${ }^{27,28}$ When the tumor volume reached about 150 $200 \mathrm{~mm}^{3}$, the tumor-bearing C57BL/6 mice were randomly assigned to three groups (each group contained six animals): group 1 was IV administrated physiological saline as a control, group 2 was IV administrated SSL-DOX ( $3 \mathrm{mg} / \mathrm{kg}$, IV, q3d [every 3 days for three doses]), and groups 3 was IV administrated iRGD-SSL-DOX (3 mg/kg, IV, q3d). Throughout the study, mice were weighed and tumors were measured with a caliper twice per week. Tumor volumes were calculated using the following formula: $\mathrm{V}=$ length $(\mathrm{cm}) \times$ width $\left(\mathrm{cm}^{2}\right) \times 0.5236$. The survival time was calculated from the 
day of B16-F10 cell inoculation (day 0) to the day of death. Kaplan-Meier survival curves were plotted for each group.

\section{Statistical analysis}

All data are shown as the mean \pm standard deviation (SD). One-way analysis of variance (ANOVA) was used to determine significance among groups, after which posthoc tests with the Bonferroni correction were used to compare between individual groups. Statistical significance was set at $P<0.05$.

\section{Results}

\section{Preparation of iRGD-SSL-DOX}

As shown in Figure 1A, iRGD was connected to the terminal of PEG through a reaction between the maleimide group of PEG and the cysteine sulfhydryl group of the iRGD peptides, through a nucleophilic addition reaction. The MALDI (matrix-assisted laser desorption/ionization) - TOF (timeof-flight mass spectrometer) - MS (mass spectrometry) results indicated that $\mathrm{R}$ RD was successfully conjugated with DSPE-PEG-maleimide (data not shown). The procedure of iRGD-SSL-DOX preparation is illustrated in Figure 1B.

\section{Characterization of iRGD-SSL-DOX}

As shown in Table 1, the average particle size of iRGD-SSLDOX was about $91 \mathrm{~nm} \pm 0.8 \mathrm{~nm}$, with a polydispersity index (PDI) of $0.16 \pm 0.003$. The zeta potential of $i R G D-S S L-D O X$ was slightly negative. The entrapment efficiency of iRGDSSL-DOX indicated that DOX was almost entrapped within the liposomes. Figure 2 shows the typical particle size and distribution of iRGD-SSL-DOX.

\section{In vitro leakage of DOX from iRGD-SSL- DOX}

The in vitro leakage of DOX from iRGD-SSL-DOX is shown in Figure 3. For iRGD-SSL-DOX groups, the leakage of DOX at the 24-hour time point was only $7.5 \%$, showing the stability of the iRGD-SSL-DOX; even at 120 hours,

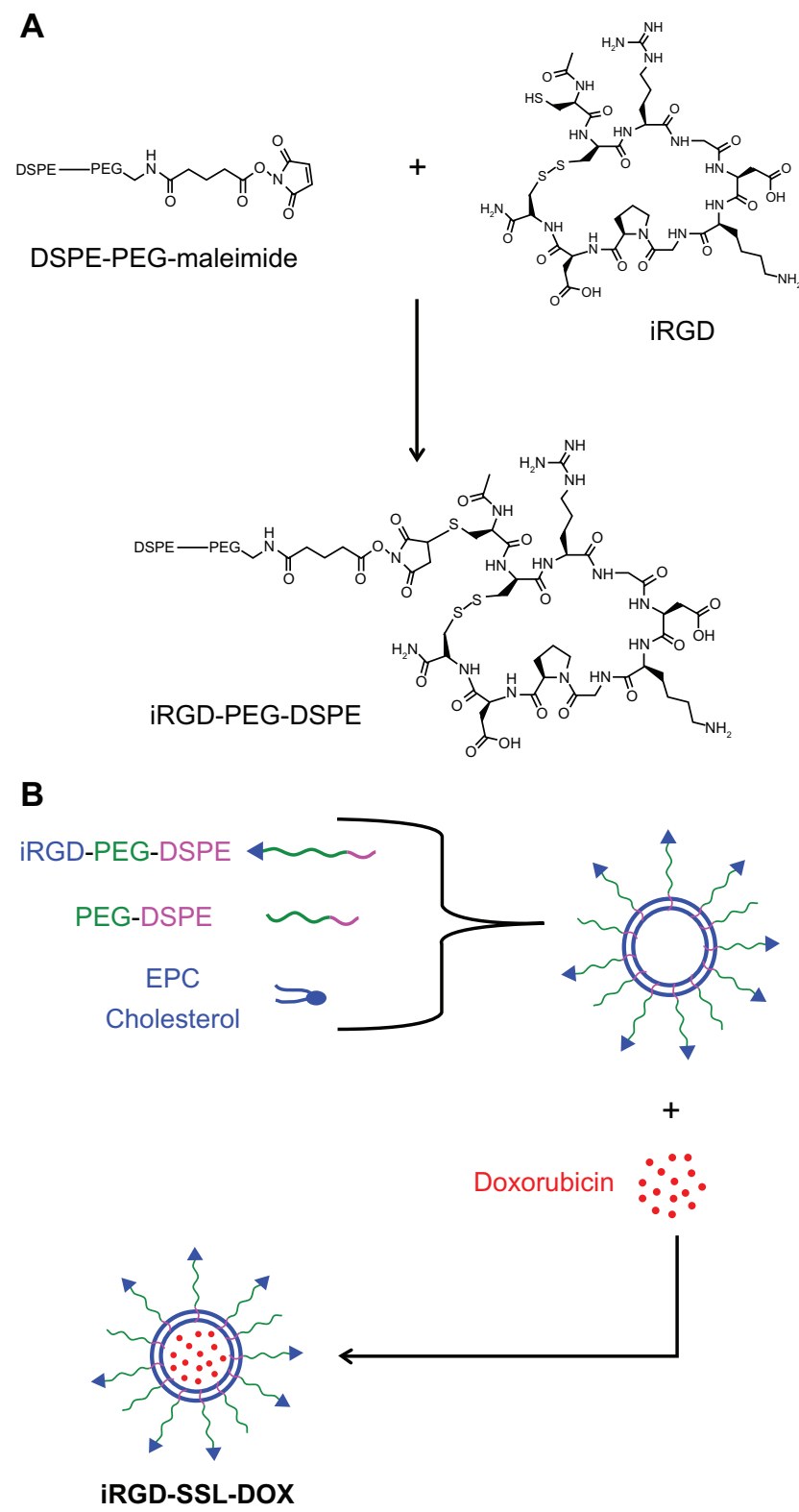

Figure I The preparation of IRGD-SSL-DOX.

Note: (A)The synthesis of iRGD-PEG-DSPE and (B) the preparation of iRGDSSL-DOX.

Abbreviations: iRGD-SSL-DOX, doxorubicin-loaded iRGD-modified sterically-stabilized liposome; iRGD, tumor-homing peptide; SSL, stericallystabilized liposome; DOX, doxorubicin; DSPE-PEG-MAL, I,2-Distearoyl-snGlycero-3-Phosphoethanolamine-N-[Maleimide(polyethylene-Glycol)-2000; PEG, polyethylene glycol; DSPE, I,2-Distearoyl-sn-glycero-3-phosphoethanolamine; EPC, egg phosphatidylcholine.

Table I The characteristics of iRGD-SSL-DOX $(n=3)$

\begin{tabular}{lllll}
\hline & $\begin{array}{l}\text { Average particle } \\
\text { size }(\mathbf{n m})\end{array}$ & Polydispersity & $\begin{array}{l}\text { Zeta potential } \\
(\mathbf{m V})\end{array}$ & $\begin{array}{l}\text { Entrapment } \\
\text { efficiency }(\%)\end{array}$ \\
\hline SSL mean \pm SD & $90.61 \pm 0.65$ & $0.18 \pm 0.001$ & $-12.30 \pm 0.20$ & $/$ \\
SSL-DOX mean \pm SD & $90.35 \pm 0.45$ & $0.17 \pm 0.002$ & $-12.23 \pm 0.11$ & $99.03 \pm 0.47$ \\
iRGD-SSL-DOX mean \pm SD & $90.74 \pm 0.75$ & $0.16 \pm 0.003$ & $-14.86 \pm 0.12$ & $98.36 \pm 0.11$ \\
\hline
\end{tabular}

Abbreviations: iRGD-SSL-DOX, doxorubicin-loaded iRGD-modified sterically-stabilized liposome; iRGD, tumor-homing peptide; SSL, sterically-stabilized liposome; DOX, doxorubicin; SD, standard deviation. 

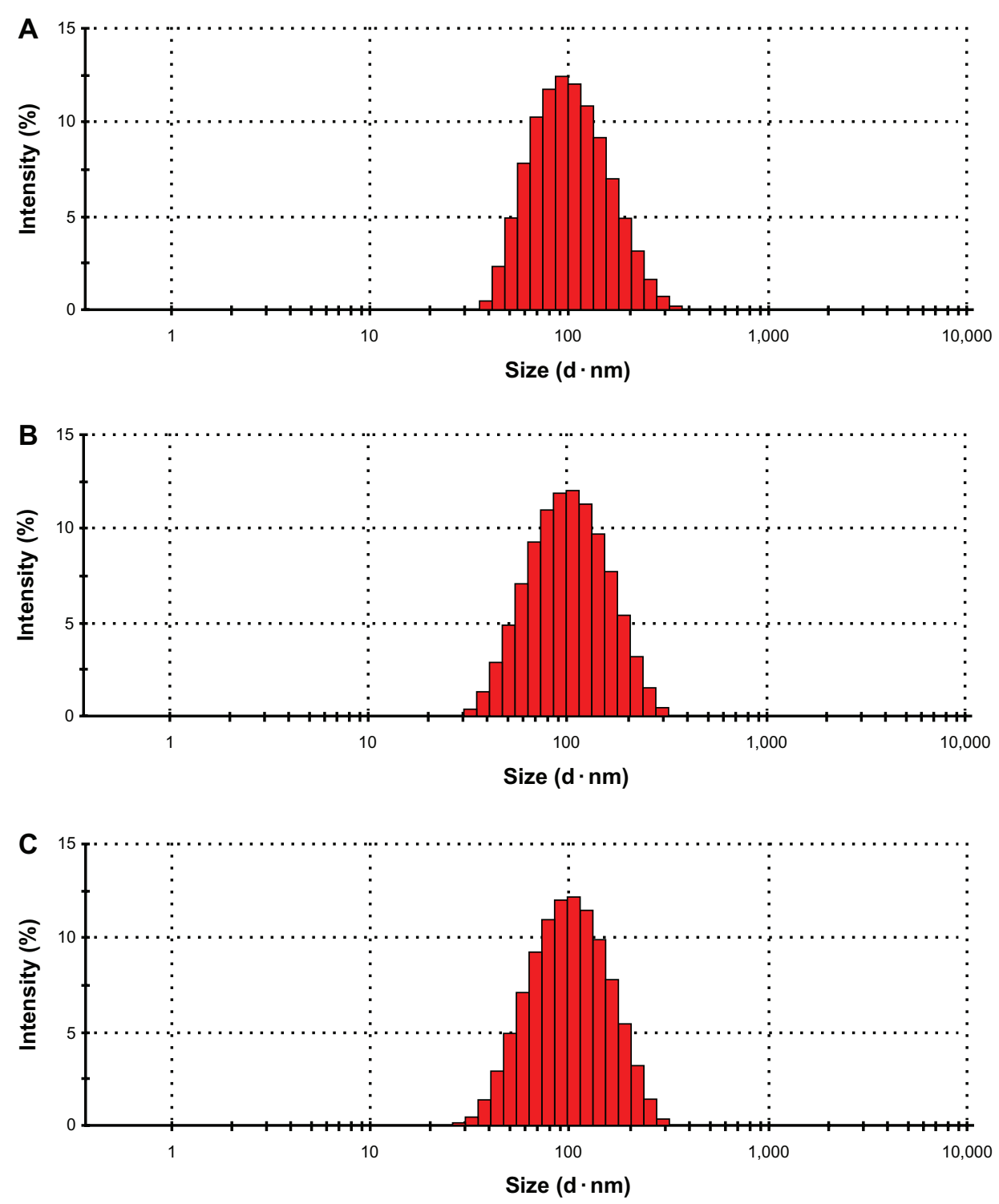

Figure 2 The typical particle size and distribution of SSL, SSL-DOX, and iRGD-SSL-DOX.

Notes: The typical particle size and distribution of (A) SSL, (B) SSL-DOX, and (C) iRGD-SSL-DOX.

Abbreviations: SSL, sterically-stabilized liposome; SSL-DOX, sterically-stabilized liposome containing DOX; DOX, doxorubicin; iRGD-SSL-DOX, DOX-loaded iRGDmodified SSL; iRGD, tumor-homing peptide.

the leakage of DOX form iRGD-SSL-DOX was only about $30 \%$. In addition, the leakage behavior of DOX from SSLDOX was similar to that of iRGD-SSL-DOX.

\section{Flow cytometry analysis}

The total coumarin-6 uptake by B16-F10 or MCF-7 cells for coumarin-6 formulations was quantified by flow cytometry determination. Without trypsin treatment groups, the cellular coumarin-6 fluorescence intensity for iRGD-SSLcoumarin-6 in B16-F10 cells was about 2.5-fold higher than that of SSL-coumarin-6 (Figure 4A). For trypsin treatment groups, as shown in Figure 4B, the cellular coumarin-6 fluorescence intensity for iRGD-SSL-coumarin-6 in B16-F10 cells was about threefold higher than that of SSL-coumarin-6 (Figure 4B). In addition, the cellular coumarin-6 level for iRGD-SSL-coumarin- 6 with or without trypsin treatment in MCF-7 cells was about 1.8- or 2.3-fold higher than that for SSL-coumarin-6, respectively (Figure 4C and D).

\section{Confocal microscopy studies}

The confocal microscopic images of B16-F10 cells after 2 hours incubation with coumarin- 6 formulations are shown in Figure 5. For the iRGD-SSL-coumarin-6 treatment group, the images show a more intense fluorescence of coumarin-6 (Figure 5B) compared with the fluorescence found in the SSL-coumarin-6 treatment group 


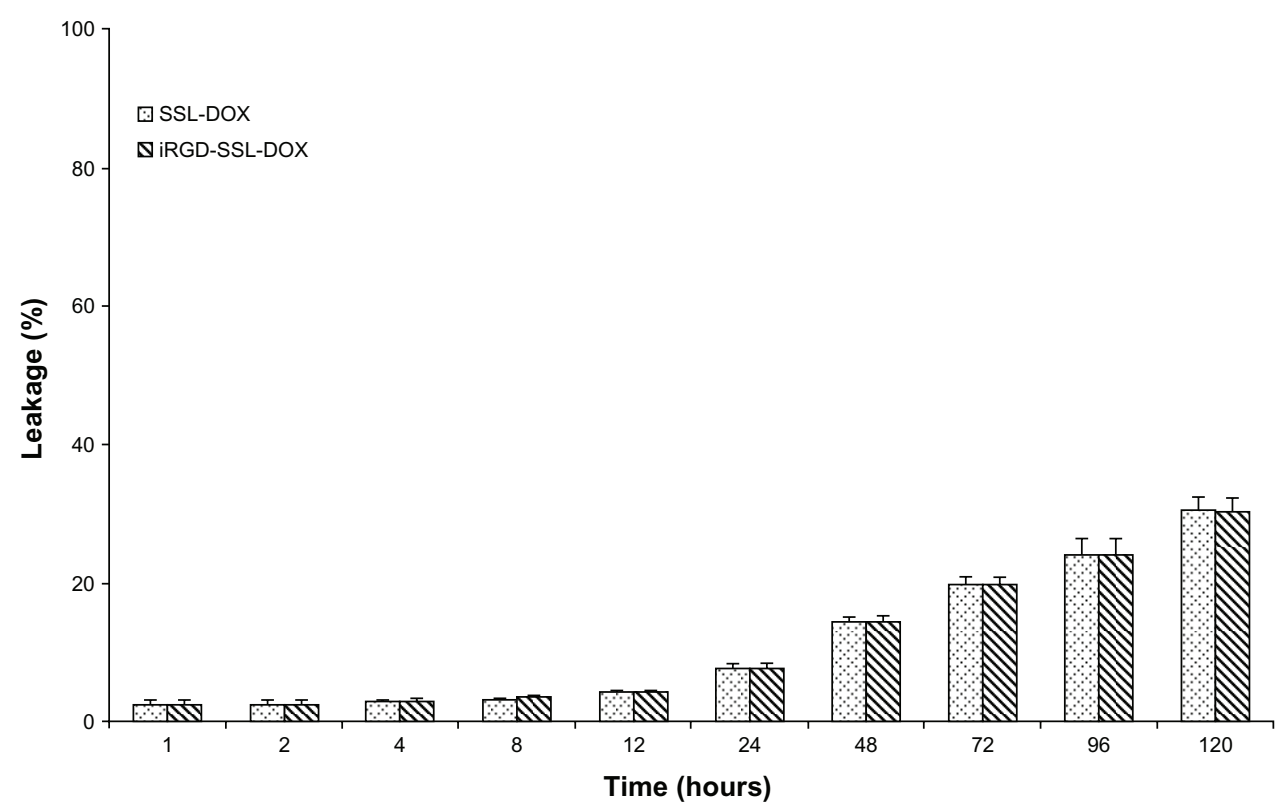

Figure 3 The leakage of DOX from iRGD-SSL-DOX.

Notes: The medium was PBS $(\mathrm{pH}=7.4)$. The temperature was controlled at $37^{\circ} \mathrm{C}$. Each datum exhibits the mean $\pm S D(n=3)$.

Abbreviations: DOX, doxorubicin; iRGD-SSL-DOX, DOX-loaded iRGD-modified SSL; SSL, sterically-stabilized liposome; PBS, phosphate buffered saline; SD, standard deviation.

A

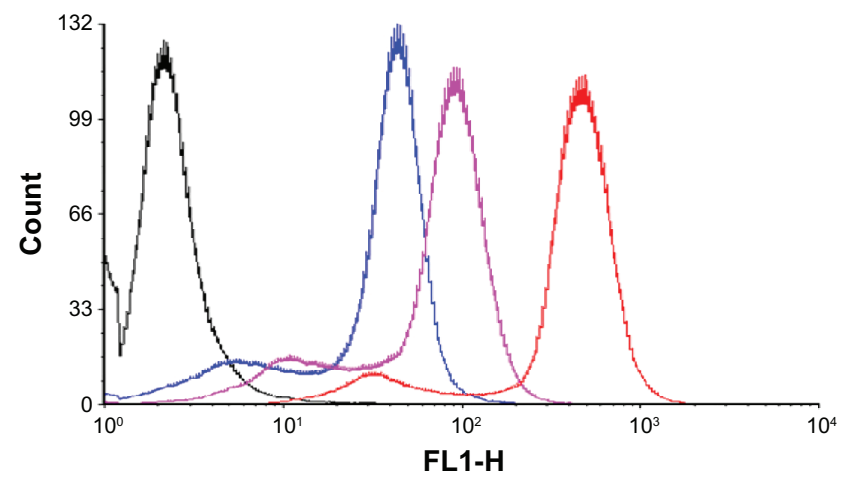

B

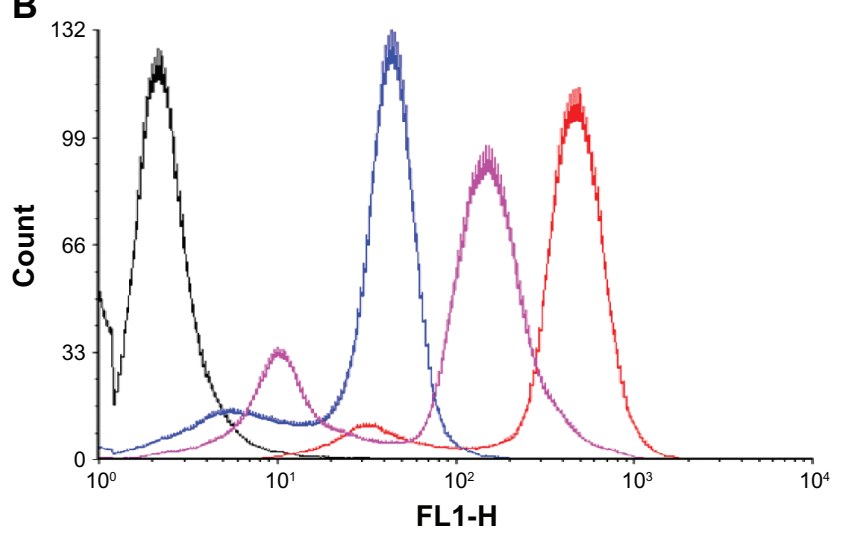

C

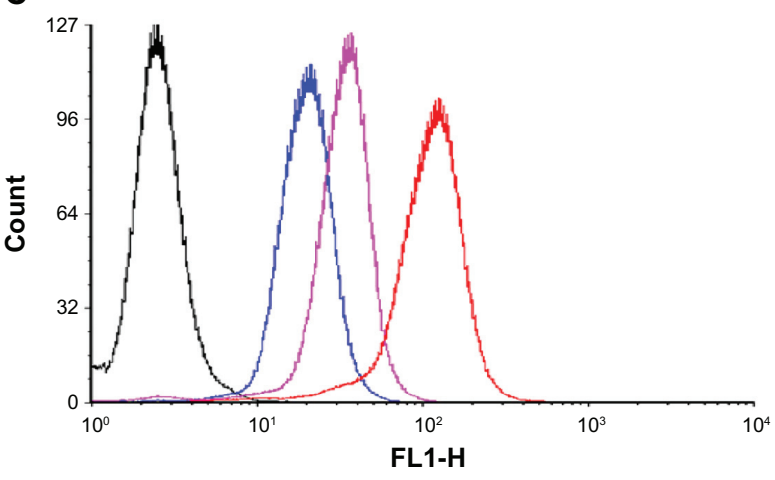

D

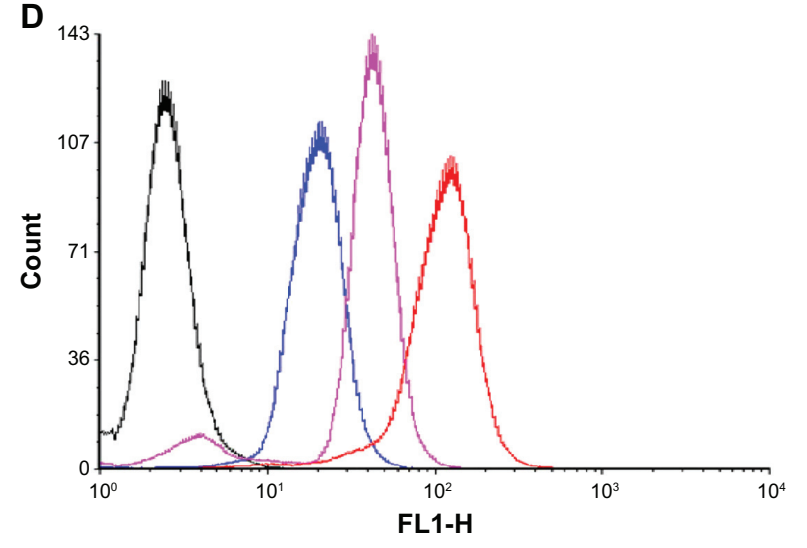

Figure 4 The flow cytometric measurement of coumarin-6 uptake from SSL-coumarin-6 or iRGD-SSL-coumarin-6 by BI6-FI0 cells and MCF-7 cells, with or without trypsin treatment, at the 2 hours incubation time point.

Notes: (A) BI6-FI0 cells, without trypsin treatment groups. (B) BI6-FI0 cells, with trypsin treatment groups. (C) MCF-7 cells, without trypsin treatment groups. (D) MCF-7 cells, with trypsin treatment groups. Black exhibits control, blue exhibits incubation with SSL-coumarin-6, pink exhibits incubation with iRGD-SSL-coumarin-6, and red exhibits coumarin-6.

Abbreviations: SSL, sterically-stabilized liposome; iRGD, tumor-homing peptide; MCF-7, breast cancer cell line. 

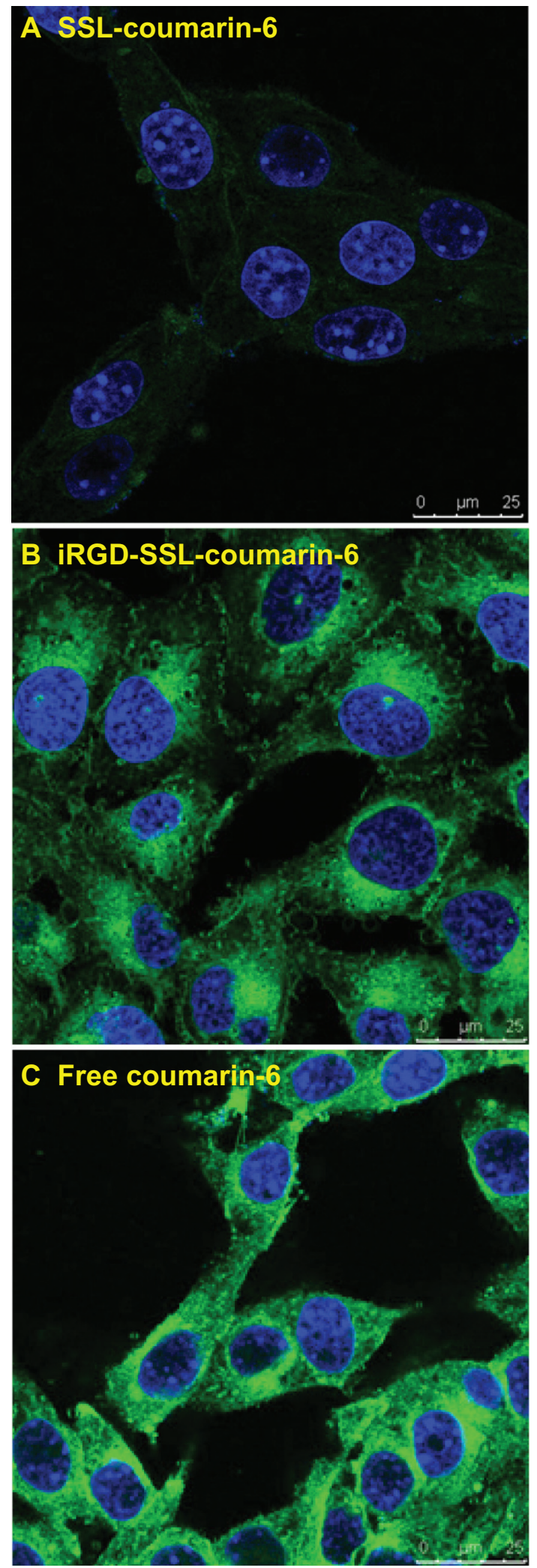

Figure 5 The confocal microscopy images of BI6-FI0 cells incubated with (A) SSLcoumarin-6, (B) iRGD-SSL-coumarin-6 or (C) free coumarin-6, for 2 hours at $37^{\circ} \mathrm{C}$. Fluorescence of coumarin- 6 exhibits green; fluorescence of Hoechst 33258 exhibits blue.

Abbreviations: iRGD, tumor-homing peptide; SSL, sterically-stabilized liposome.
(Figure 5A). As shown in Figure 5C, free coumarin-6 readily partitioned into the lipid membranes and then diffused into the three types of cells, leading to a greater cellular accumulation due to its highly hydrophobic nature. Therefore, free coumarin- 6 was selected as the positive control group.

\section{Biodistribution of DiR-loaded liposomes in tumor-bearing nude mice}

The distribution and tumor accumulation of fluorescent DiR in B16-F10 tumor-bearing nude mice is shown in Figure 6. Compared with control group, the DiR fluorescence signal in tumor site was observed in SSL-DiR treatment and iRGDSSL-DiR treatment groups from the 1-hour to the 24-hour time point. The fluorescence signal of $\mathrm{DiR}$ in the tumor site was stronger in the iRGD-SSL-DiR treatment group than that found in the SSL-DiR treatment group, at all observed time points.

\section{Immunohistochemistry study}

In the immunohistochemistry study, the nuclei stained with Hoechst 33258 are represented by a blue fluorescence (Figure 7A1 and B1), the blood vessels stained with CD31 are represented by a green fluorescence (Figure 7A2 and B2), while DiI is represented by a red fluorescence (Figure 7A3 and B3). At the 3-hour time point after administration, iRGDSSL-DiI mostly penetrated from the tumor blood vessels and gradually accumulated to tumor cells (Figure 7B4), while SSL-DiI still existed in the tumor blood vessels (Figure 7A4). In addition, it could also be observed that the red fluorescence intensity of DiI in the iRGD-SSL-DiI treatment group was stronger than that of the SSL-DiI treatment group.

\section{In vivo antitumor activity of iRGD-SSL- DOX}

The antitumor effect of iRGD-SSL-DOX was evaluated in B16-F10 tumor-bearing C57BL/6 mice. The tumor growth was significantly inhibited in SSL-DOX and iRGD-SSLDOX treatment groups compared with the physiological saline treatment group $(P<0.01)$, as shown in Figure 8 . iRGD-SSL-DOX significantly inhibited the growth of B16F10 tumors compared with that in the SSL-DOX treatment groups $(P<0.01)$. The average tumor size at day 22 in the SSL-DOX and iRGD-SSL-DOX group was $3,310 \mathrm{~mm}^{3}$ $\pm 1,022 \mathrm{~mm}^{3}$ and $1205 \mathrm{~mm}^{3} \pm 496 \mathrm{~mm}^{3}$, respectively, compared with $6,975 \mathrm{~mm}^{3} \pm 1,100 \mathrm{~mm}^{3}$ in the physiological saline group $(P<0.01)$. The corresponding tumor growth 


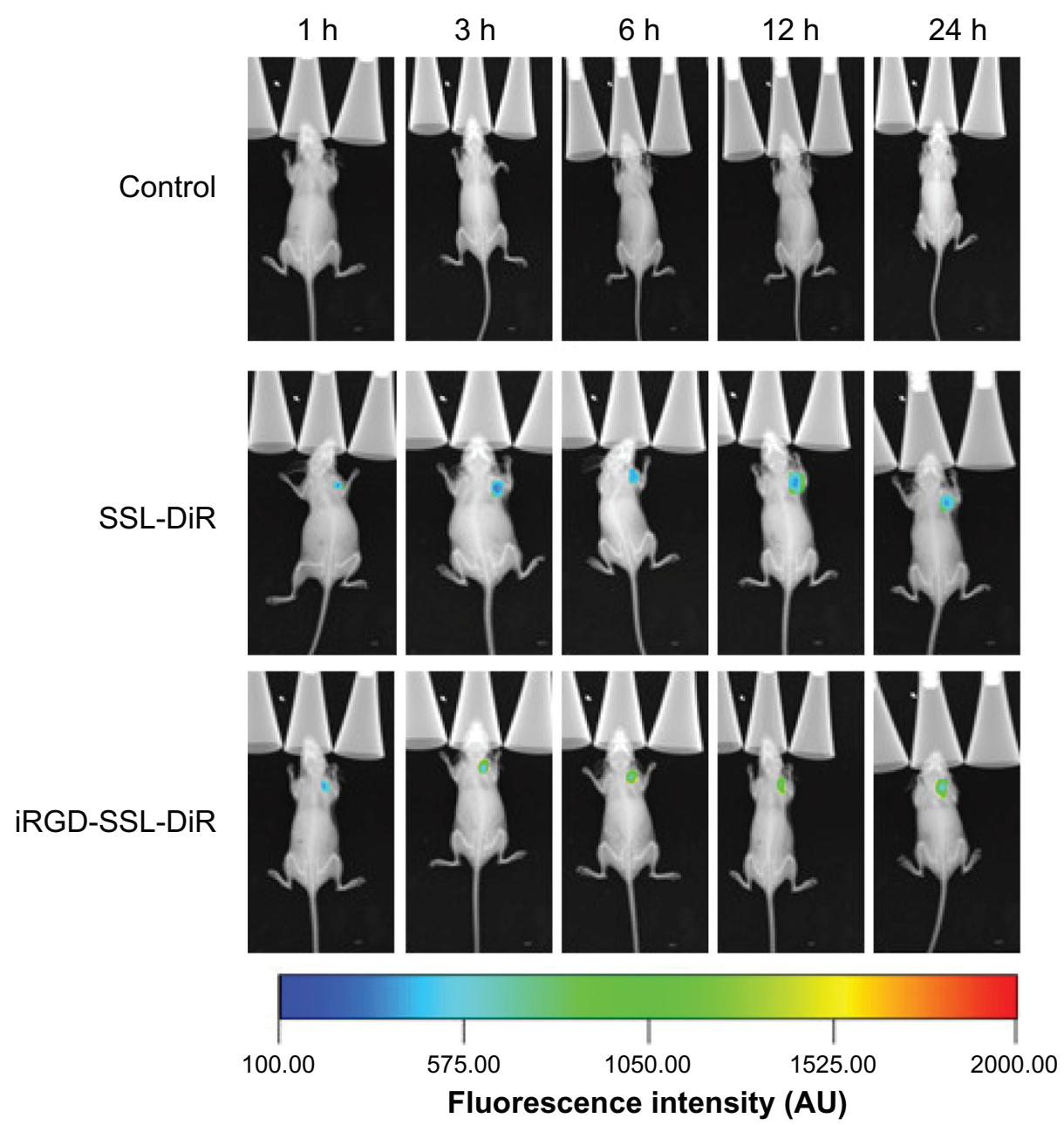

Figure 6 In vivo image of biodistribution of iRGD-SSL-DiR in BI6-FI0 tumor-bearing nude mice.

Note: In vivo whole body imaging of BI6-FI0 tumor-bearing nude mice after IV administered physiological saline (as control), SSL-DiR, and iRGD-SSL-DiR, respectively. Abbreviations: SSL, sterically-stabilized liposome; DiR, carbocyanine dye I,I'-dioctadecyltetramethyl indotricarbocyanine iodide; iRGD, tumor-homing peptide; IV, intravenous.

inhibition in the SSL-DOX and iRGD-SSL-DOX treated groups was $52.5 \%$ and $82.7 \%$, respectively. The KaplanMeier survival curve is represented in Figure 9. After administration three times, the median survival time of mice treated with iRGD-SSL-DOX (43.5 days) was significantly longer than that of mice treated with physiological saline (27 days, $P<0.01)$ and SSL-DOX (33 days, $P<0.01$ ), respectively.

\section{Discussion}

iRGD is a tumor-targeting and tumor-penetrating peptide which can enhance the permeability of tumor cells mediated by integrins and NRP-1 which upregulate tumor cells. ${ }^{11,12,29}$ Considering the mechanism of iRGD, in the present study, we designed and prepared a iRGD-SSL-DOX and suggested that the iRGD in the iRGD-SSL-DOX bound firstly with $\alpha v$ integrins and then with NRP-1, which can mediate the delivery system penetrating into the tumor cells. The possible role of iRGD-SSL-DOX in tumor-targeting and tumor-penetrating activities is illustrated in Figure 10.

Integrins are central regulators in multicellular biology. Many human pathologies (including inflammation, cancer, fibrosis, and infectious diseases) involve integrin adhesion. ${ }^{30}$ Integrins play a direct role in tumor progression, specifically in tumor cell survival, tumor angiogenesis, and metastasis. ${ }^{31}$ $\alpha \mathrm{v}$ integrins are highly expressed on endothelial cells and tumor cells, and could bind with the RGD peptide sequence. ${ }^{32}$ The neuropilin receptors were first discovered as regulators of nervous system development and then identified as receptors for vascular endothelial growth factor. ${ }^{33}$ It has been reported that NRP-1 is overexpressed on several types of tumor cells, such as prostate cancer, colorectal cancer, lung cancer, breast cancer, and astrocytomas. ${ }^{34}$ Considering both overexpression of $\alpha v$ integrin receptor and NRP-1 in B16-F10 cells, ${ }^{24,25}$ in 

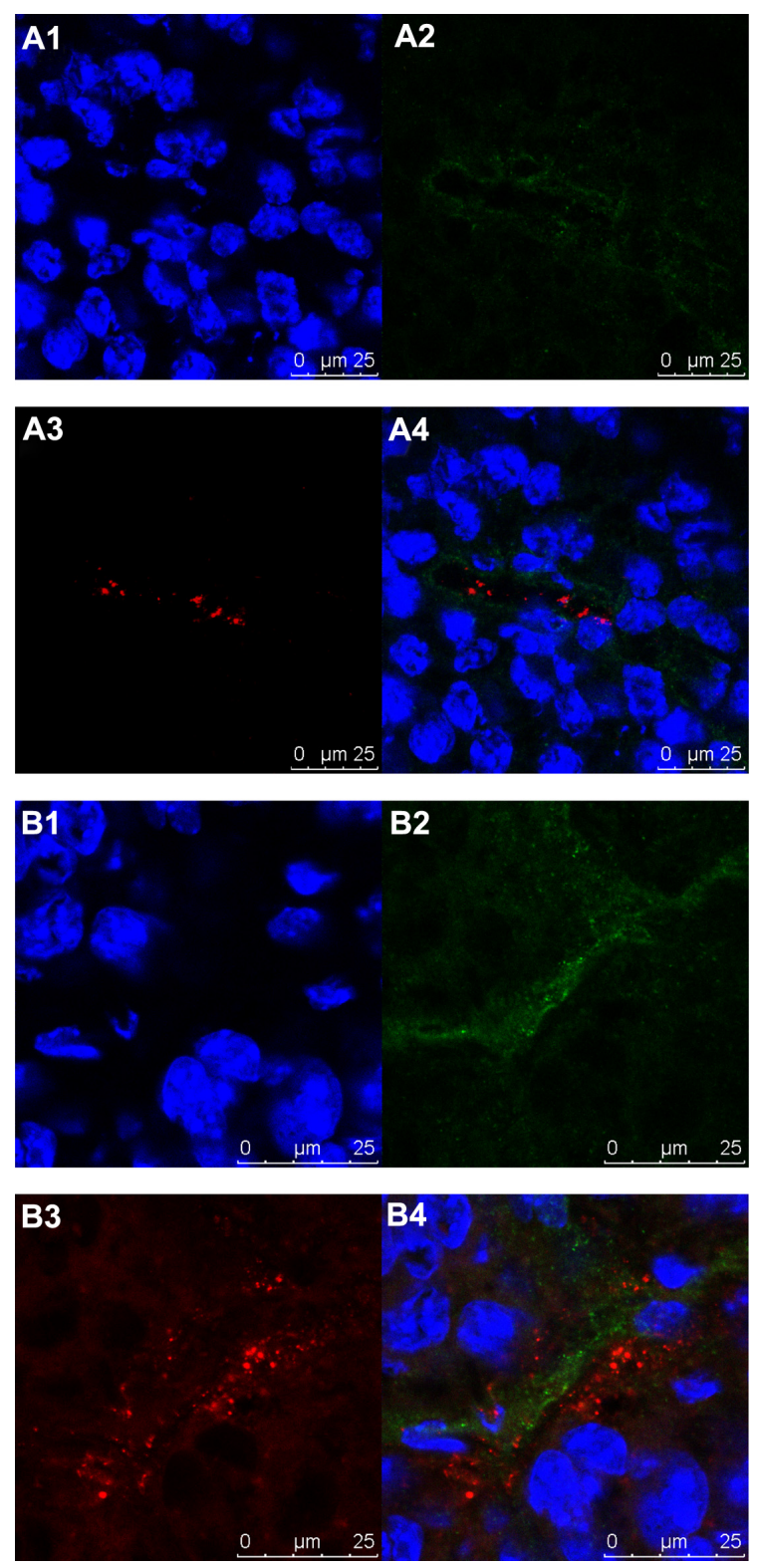

Figure 7 The localization of iRGD-SSL-Dil in tumor section.

Notes: BI6-FI0 tumor-bearing nude mice were IV administered with SSL-Dil or iRGD-SSL-Dil at a dose of $1200 \mathrm{ng} /$ animal. At the 3-hour time point, the mice were sacrificed, and the tumors were harvested. The tumor sections were immune-stained and examined for fluorescence. Images in cluster represent (A) SSL-Dil treatment group, and (B) iRGD-SSL-Dil treatment group. Nuclei were counterstained with Hoechst 33258 (blue; AI and BI). Tumor blood vessels represented a green fluorescence (antiCD31, green; A2 and B2). Dil represented a red fluorescence (red; A3 and B3). Merge (A4 and B4).

Abbreviations: iRGD, tumor-homing peptide; SSL, sterically-stabilized liposome; Dil, fluorescent probe, I, I'-dioctadecyl-3,3,3',3'-tetramethylindocarbocyanine perchlorate; IV, intravenous.

the present study, we selected the B16-F10 cells as a model for tumor cells so as to investigate the antitumor activity of iRGD-SSL-DOX in vitro and in vivo.

The targeting effect of the iRGD-modified SSL on B16-F10 cells was observed in our flow cytometry and confocal experiments (Figures 4A and 5); this effect was due to iRGD recognizing the $\alpha$ v integrin receptors with the RGD motif. The in vivo biodistribution results also demonstrated the tumor-targeting effect of the iRGD-modified SSL (Figure 6). When iRGD-SSL-coumarin-6 was treated with trypsin, the coumarin-6 fluorescence-intensity ratio of iRGD-SSL-coumarin-6/SSL-coumarin-6 was further increased, compared with the ratio for the treatment groups without trypsin (Figure 4A and C). We suggest that the iRGD might partly proteolytically cleave to produce the CRGDK fragment, when iRGD-SSL-coumarin-6 was treated with trypsin, which has the affinity for NRP-1, to trigger the penetration. Interestingly, we also observed that the coumarin-6 fluorescence intensity for iRGD-SSLcoumarin-6 (with or without trypsin treatment) was also higher than that for SSL-coumarin-6 (Figure 4C and D) in $\alpha \mathrm{v}$ integrin-negative MCF-7 cells. ${ }^{35,36}$ We suggest that this is due to the expression of NRP-1 in the MCF-7 cells. ${ }^{37}$ When iRGD-SSL-coumarin- 6 or iRGD-SSL-coumarin- 6 treated with trypsin was incubated with MCF-7 cells, the iRGD might partly proteolytic cleave to produce the CRGDK fragment which has the necessary affinity for NRP-1 to trigger the penetration. These above results might partly explain the tumor-penetrating action of the iRGD, which proteolytically cleaved to produce the CRGDK fragment mediated with NRP-1. In addition, our immunohistochemistry study indicated that the iRGD-SSL-DiI mostly penetrated from the tumor blood vessels, and gradually accumulated to tumor cells, compared with the results for SSL-DiI (Figure 7B4 and $\mathrm{A} 4$, respectively).

Considering the EPR effect, as well as ligand-modified SSL as an active-targeting delivery system, ${ }^{38-40}$ we selected iRGD as a ligand and developed a novel iRGD-modified SSL. Our particle size, distribution, and in vitro leakage results indicated that the prepared iRGD-SSL-DOX is desirable.

Because the antitumor activity of the DOX delivery system on melanoma has been demonstrated, ${ }^{27,41-43}$ in the present study we selected DOX as a model drug to prepare the iRGD-SSL-DOX. The antitumor activity of iRGD-SSLDOX was evaluated in a B16-F10 bearing animal model. In addition, because the antitumor activity of free DOX in a B16-F10 bearing animal model is limited, ${ }^{43}$ in the present in vivo antitumor activity study we did select free DOX as a treatment group. Our in vivo antitumor activity results indicated iRGD could be used as a tumor-targeting and tumor-penetrating ligand for tumor targeting drug delivery systems. 


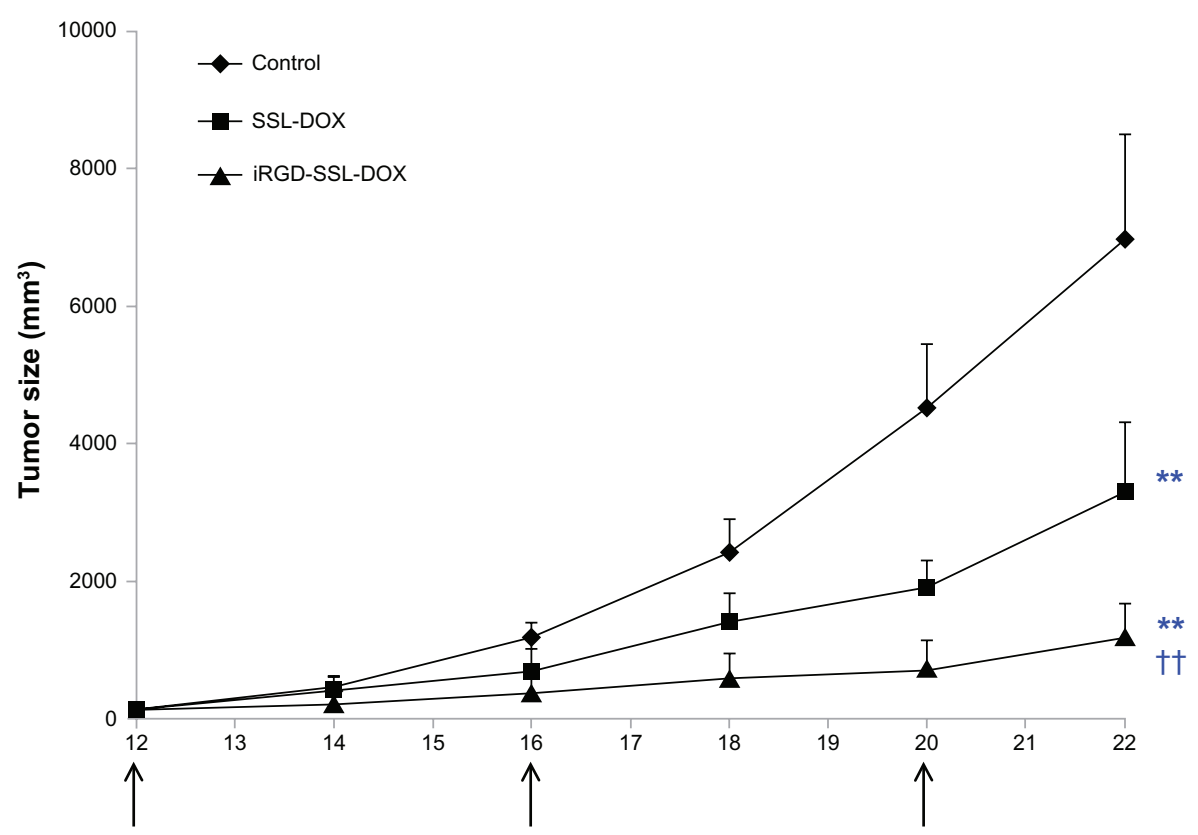

Days after incubation (day)

Figure 8 In vivo antitumor activity of iRGD-SSL-DOX.

Notes: C57BL/6 mice were inoculated SC with BI6-FI0 cells and treated with physiological saline, SSL-DOX (3 mg/kg, IV, q3d), iRGD-SSL-DOX (3 mg/kg, IV, q3d). The formulations were given via the tail vein for all administrations. The tumors were measured with a caliper twice per week throughout the study. $* * P<0.0 \mathrm{I}$ versus physiological saline as control; ${ }^{\dagger \dagger} P<0.01$ versus SSL-DOX treatment group. $\uparrow$, administration.

Abbreviations: iRGD-SSL-DOX, doxorubicin-loaded iRGD-modified sterically-stabilized liposome; iRGD, tumor-homing peptide; SSL, sterically-stabilized liposome; DOX, doxorubicin; SC, subcutaneous; IV, intravenous; q3d, every 3 days for three doses.

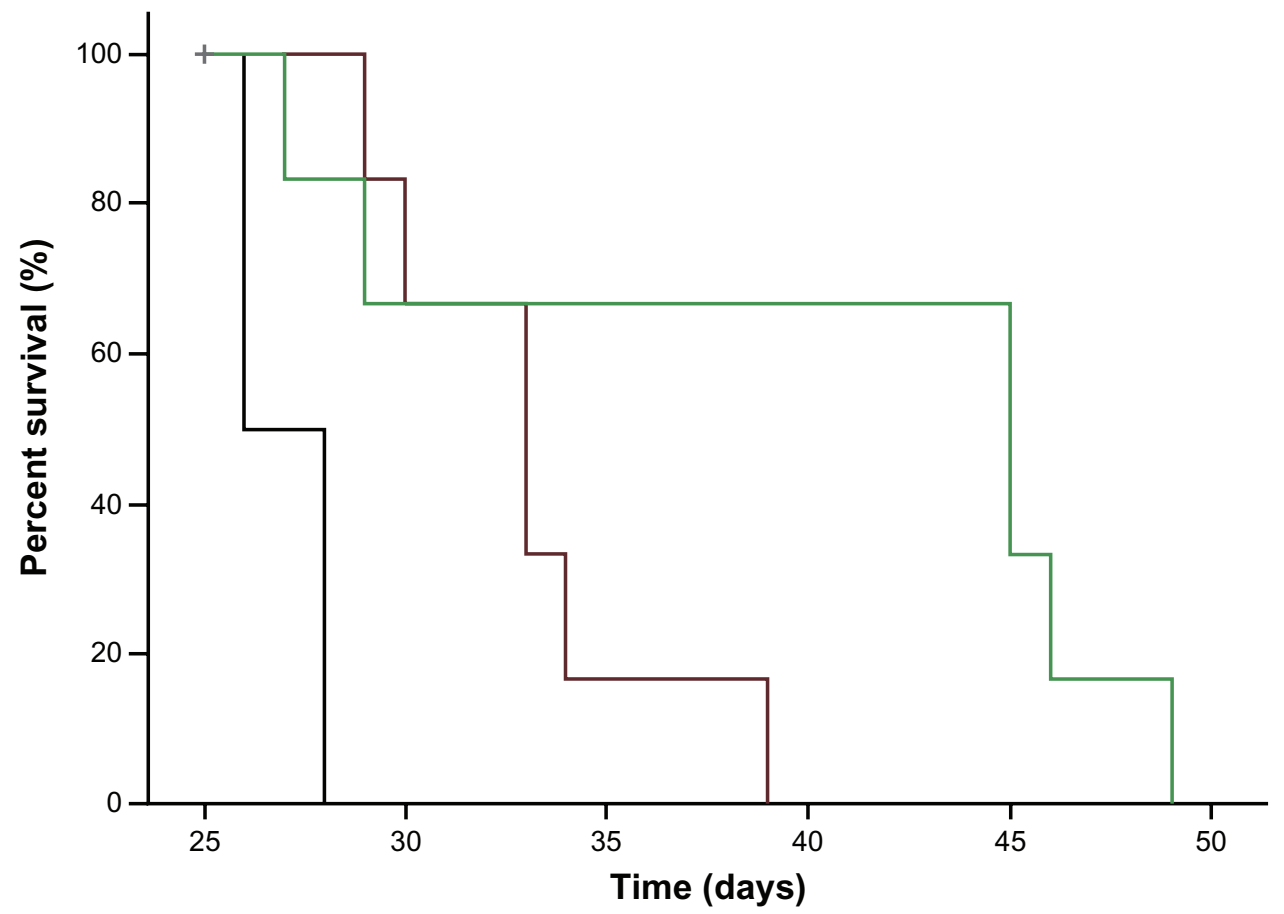

Figure 9 Kaplan-Meier survival curves of BI6-FI0 tumor-bearing C57BL/6 mice treated with iRGD-SSL-DOX.

Notes: Kaplan-Meier survival curves of BI6-FIO tumor-bearing C57BL/6 mice treated with physiological saline (black), SSL-DOX (3 mg/kg, IV, q3d) (brown), iRGD-SSLDOX (3 mg/kg, IV, q3d) (green). Results indicated that the iRGD-SSL-DOX (43.5 days) significantly improved the median survival time of mice as compared with that treated with SSL-DOX (33 days, $P<0.0 \mathrm{I})$ and physiological saline $(27$ days, $P<0.0 \mathrm{I})$, respectively.

Abbreviations: iRGD-SSL-DOX, doxorubicin-loaded iRGD-modified sterically-stabilized liposome; iRGD, tumor-homing peptide; SSL, sterically-stabilized liposome; DOX, doxorubicin; IV, intravenous; q3d, every 3 days for three doses. 


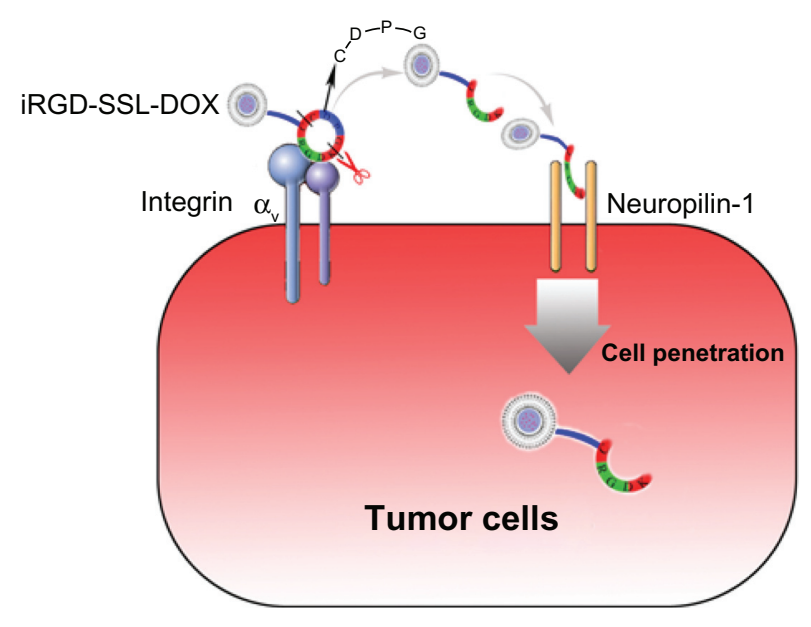

Figure 10 The detailed scheme of tumor-targeting and tumor-penetrating effect of iRGD-SSL-DOX.

Note: The RGD motif in iRGD-SSL-DOX mediates binding to $\alpha v$ integrins firstly on tumor cells followed by a proteolytic cleavage, exposing a binding motif of CRGDK sequences for NRP-I, which can then mediate penetration into tumor cells. Abbreviations: iRGD-SSL-DOX, doxorubicin-loaded iRGD-modified stericallystabilized liposome; iRGD, tumor-homing peptide; SSL, sterically-stabilized liposome; DOX, doxorubicin; CRGDK, Cys-Arg-Gly-Asp-Lys.

\section{Conclusion}

In the present study, we prepared a novel iRGD-SSL-DOX with the aim of evaluating its antitumor activity on B16F10 melanoma cells, in vitro and in vivo. The tumor-targeting and tumor-penetrating activity of the iRGD-modified SSL was demonstrated by in vitro flow cytometry and confocal microscopy, as well as in vivo biodistribution and confocal immunofluorescence microscopy experiments. The antitumor activity of the iRGD-SSL-DOX against melanoma tumors was confirmed in our in vivo B16-F10 tumor-bearing mice. The iRGD-SSL-DOX is a tumor-targeting and tumorpenetrating peptide modified liposome which has significant antitumor activity against melanoma tumors.

\section{Acknowledgments}

The authors gratefully acknowledge the financial support from the National Natural Science Foundation of China (No 81172992), the National Basic Research Program of China (973 Program 2009CB930300 and 2013CB932501), and the Innovation Team of the Ministry of Education of China (No BMU20110263).

\section{Disclosure}

The authors report no conflicts of interest in this work.

\section{References}

1. Lammers T, Kiessling F, Hennink WE, Storm G. Drug targeting to tumors: principles, pitfalls and (pre-) clinical progress. J Control Release. 2012;161(2):175-187.
2. Valencia PM, Hanewich-Hollatz MH, Gao W, et al. Effects of ligands with different water solubilities on self-assembly and properties of targeted nanoparticles. Biomaterials. 2011;32(26):6226-6233.

3. Xiao Z, Levy-Nissenbaum E, Alexis F, et al. Engineering of targeted nanoparticles for cancer therapy using internalizing aptamers isolated by cell-uptake selection. ACS Nano. 2012;6(1):696-704.

4. Wang W, Cheng D, Gong F, Miao X, Shuai X. Design of multifunctional micelle for tumor-targeted intracellular drug release and fluorescent imaging. Adv Mater. 2012;24(1):115-120.

5. Eldar-Boock A, Miller K, Sanchis J, Lupu R, Vicent MJ, Satchi-Fainaro R. Integrin-assisted drug delivery of nano-scaled polymer therapeutics bearing paclitaxel. Biomaterials. 2011;32(15):3862-3874.

6. Yang Z, Luo X, Zhang X, Liu J, Jiang Q. Targeted delivery of 10-hydroxycamptothecin to human breast cancers by cyclic RGDmodified lipid-polymer hybrid nanoparticles. Biomed Mater. 2013;8(2): 025012 .

7. Chen Z, Deng J, Zhao Y, Tao T. Cyclic RGD peptide-modified liposomal drug delivery system: enhanced cellular uptake in vitro and improved pharmacokinetics in rats. Int J Nanomedicine. 2012;7: 3803-3811.

8. Cai LL, Liu P, Li X, et al. RGD peptide-mediated chitosan-based polymeric micelles targeting delivery for integrin-overexpressing tumor cells. Int J Nanomedicine. 2011;6:3499-3508.

9. Zhao BJ, Ke XY, Huang Y, et al. The antiangiogenic efficacy of NGR-modified PEG-DSPE micelles containing paclitaxel (NGR-MPTX) for the treatment of glioma in rats. J Drug Target. 2011;19(5): 382-390.

10. Luo LM, Huang Y, Zhao BX, et al. Anti-tumor and anti-angiogenic effect of metronomic cyclic NGR-modified liposomes containing paclitaxel. Biomaterials. 2013;34(4):1102-1114.

11. Sugahara KN, Teesalu T, Karmali PP, et al. Tissue-penetrating delivery of compounds and nanoparticles into tumors. Cancer Cell. 2009;16(6):510-520.

12. Sugahara KN, Teesalu T, Karmali PP, et al. Coadministration of a tumor-penetrating peptide enhances the efficacy of cancer drugs. Science. 2010;328(5981):1031-1035.

13. Zhu Z, Xie C, Liu Q, et al. The effect of hydrophilic chain length and iRGD on drug delivery from poly( $\varepsilon$-caprolactone)-poly( $\mathrm{N}$-vinylpyrrolidone) nanoparticles. Biomaterials. 2011;32(35):9525-9535.

14. Song W, Li M, Tang Z, et al. Methoxypoly(ethylene glycol)-blockpoly(L-glutamic acid)-loaded cisplatin and a combination with iRGD for the treatment of non-small-cell lung cancers. Macromol Biosci. 2012;12(11):1514-1523.

15. Wang X, Zhen X, Wang J, Zhang J, Wu W, Jiang X. Doxorubicin delivery to 3D multicellular spheroids and tumors based on boronic acid-rich chitosan nanoparticles. Biomaterials. 2013;34(19):4667-4679.

16. Su S, Wang H, Liu X, Wu Y, Nie G. iRGD-coupled responsive fluorescent nanogel for targeted drug delivery. Biomaterials. 2013;34(13): $3523-3533$.

17. Hai-Tao Z, Hui-Cheng L, Zheng-Wu L, Chang-Hong G. A tumorpenetrating peptide modification enhances the antitumor activity of endostatin in vivo. Anticancer Drugs. 2011;22(5):409-415.

18. Allen TM, Cullis PR. Liposomal drug delivery systems: from concept to clinical applications. Adv Drug Deliv Rev. 2013;65(1):36-48.

19. Huwyler J, Drewe J, Krähenbuhl S. Tumor targeting using liposomal antineoplastic drugs. Int J Nanomedicine. 2008;3(1):21-29.

20. Moghimi SM, Patel HM. Opsonophagocytosis of liposomes by peritoneal macrophages and bone marrow reticuloendothelial cells. Biochim Biophys Acta. 1992;1135(3):269-274.

21. Lasic DD. Doxorubicin in sterically stabilized liposomes. Nature. 1996;380(6574):561-562.

22. Yuan F, Dellian M, Fukumura D, et al. Vascular permeability in a human tumor xenograft: molecular size dependence and cutoff size. Cancer Res. 1995;55(17):3752-3756.

23. Maruyama K. Intracellular targeting delivery of liposomal drugs to solid tumors based on EPR effects. Adv Drug Deliv Rev. 2011;63(3): 161-169. 
24. Ratheesh A, Ingle A, Gude RP. Pentoxifylline modulates cell surface integrin expression and integrin mediated adhesion of B16F10 cells to extracellular matrix components. Cancer Biol Ther. 2007;6(11): 1743-1752.

25. Mazurek AM, Olbryt M. The influence of neuropilin-1 silencing on semaphorin $3 \mathrm{~A}$ and $3 \mathrm{C}$ activity in B16(F10) murine melanoma cells. Neoplasma. 2012;59(1):43-51.

26. Zhao BX, Zhao Y, Huang Y, et al. The efficiency of tumor-specific $\mathrm{pH}$ responsive peptide-modified polymeric micelles containing paclitaxel. Biomaterials. 2012;33(8):2508-2520.

27. Yang T, Wang Y, Li Z, et al. Targeted delivery of a combination therapy consisting of combretastatin A4 and low-dose doxorubicin against tumor neovasculature. Nanomedicine. 2012;8(1):81-92.

28. Wang Y, Yang T, Wang X, et al. Materializing sequential killing of tumor vasculature and tumor cells via targeted polymeric micelle system. J Control Release. 2011;149(3):299-306.

29. Marcucci F, Corti A. How to improve exposure of tumor cells to drugs promoter drugs increase tumor uptake and penetration of effector drugs. Adv Drug Deliv Rev. 2012;64(1):53-68

30. Goodman SL, Picard M. Integrins as therapeutic targets. Trends Pharmacol Sci. 2012;33(7):405-412.

31. Nemeth JA, Nakada MT, Trikha M, et al. Alpha-v integrins as therapeutic targets in oncology. Cancer Invest. 2007;25(7):632-646.

32. Tucker GC. Alpha v integrin inhibitors and cancer therapy. Curr Opin Investig Drugs. 2003;4(6):722-731.

33. Bagri A, Tessier-Lavigne M, Watts RJ. Neuropilins in tumor biology. Clin Cancer Res. 2009;15(6):1860-1864.

34. Ellis LM. The role of neuropilins in cancer. Mol Cancer Ther. 2006;5(5): 1099-1107.
35. Cao J, Wan S, Tian J, et al. Fast clearing RGD-based near-infrared fluorescent probes for in vivo tumor diagnosis. Contrast Media $\mathrm{Mol}$ Imaging. 2012;7(4):390-402.

36. Biswas S, Wang X, Morales AR, Ahn HY, Belfield KD. Integrin-targeting block copolymer probes for two-photon fluorescence bioimaging. Biomacromolecules. 2011;12(2):441-449.

37. Nasarre P, Constantin B, Rouhaud L, et al. Semaphorin SEMA3F and VEGF have opposing effects on cell attachment and spreading. Neoplasia. 2003;5(1):83-92.

38. Vail DM, Amantea MA, Colbern GT, Martin FJ, Hilger RA, Working PK. Pegylated liposomal doxorubicin: proof of principle using preclinical animal models and pharmacokinetic studies. Semin Oncol. 2004;31(6 Suppl 13):16-35.

39. Acharya S, Sahoo SK. PLGA nanoparticles containing various anticancer agents and tumour delivery by EPR effect. Adv Drug Deliv Rev. 2011;63(3):170-183.

40. Pastorino F, Brignole C, Marimpietri D, et al. Vascular damage and antiangiogenic effects of tumor vessel-targeted liposomal chemotherapy. Cancer Res. 2003;63(21):7400-7409.

41. Al-Jamal WT, Al-Ahmady ZS, Kostarelos K. Pharmacokinetics \& tissue distribution of temperature-sensitive liposomal doxorubicin in tumor-bearing mice triggered with mild hyperthermia. Biomaterials. 2012;33(18):4608-4617.

42. Dai W, Yang T, Wang Y, et al. Peptide PHSCNK as an integrin $\alpha 5 \beta 1$ antagonist targets stealth liposomes to integrin-overexpressing melanoma. Nanomedicine. 2012;8(7):1152-1161.

43. Fan Y, Du W, He B, et al. The reduction of tumor interstitial fluid pressure by liposomal imatinib and its effect on combination therapy with liposomal doxorubicin. Biomaterials. 2013;34(9):2277-2288.
International Journal of Nanomedicine

\section{Publish your work in this journal}

The International Journal of Nanomedicine is an international, peerreviewed journal focusing on the application of nanotechnology in diagnostics, therapeutics, and drug delivery systems throughou the biomedical field. This journal is indexed on PubMed Central, MedLine, CAS, SciSearch $₫$, Current Contents $₫ /$ Clinical Medicine,

\section{Dovepress}

Journal Citation Reports/Science Edition, EMBase, Scopus and the Elsevier Bibliographic databases. The manuscript management system is completely online and includes a very quick and fair peer-review system, which is all easy to use. Visit http://www.dovepress.com/ testimonials.php to read real quotes from published authors. 